\title{
Real-world challenge for clinicians treating advanced gastroesophageal adenocarcinoma (Review)
}

\author{
MARK A. BAXTER ${ }^{1,2}$, RUSSELL D. PETTY ${ }^{1,2}$, DANIEL SWINSON ${ }^{3}$, PETER S. HALL $^{4}$ and SHANE O'HANLON $^{5}$ \\ ${ }^{1}$ Division of Molecular and Clinical Medicine, Ninewells Hospital and Medical School, University of Dundee, \\ Dundee DD2 1SY, Scotland; ${ }^{2}$ Tayside Cancer Centre, Ninewells Hospital and Medical School, NHS Tayside, \\ Dundee DD1 9SY; ${ }^{3}$ Department of Oncology, St. James's Hospital, Leeds Teaching Hospitals NHS Trust, Leeds LS9 7TF; \\ ${ }^{4}$ Edinburgh Cancer Research Centre, University of Edinburgh, Edinburgh EH4 2XR, Scotland, UK; \\ ${ }^{5}$ Department of Geriatric Medicine, St. Vincent's University Hospital, Dublin 4, D04 N2E0, Republic of Ireland
}

Received November 12, 2020; Accepted March 4, 2021

DOI: $10.3892 /$ ijo.2021.5202

\begin{abstract}
Gastroesophageal adenocarcinoma (GOA) is a disease of older people. Incidence is rising in the developed world and the majority of patients present with advanced disease. Based on clinical trial data, systemic chemotherapy in the advanced setting is associated with improvements in quality of life and survival. However, there is a recognised mismatch between trial populations and the patients encountered in clinical practice in terms of age, comorbidity and fitness. Appropriate patient selection is essential to safely deliver effective treatment. In this narrative review, we discuss the challenges faced by clinicians when assessing real-world patients with advanced GOA for systemic therapy. We also highlight the importance of frailty screening and the current available evidence we can use to guide our management.
\end{abstract}

Correspondence to: Dr Mark A. Baxter, Division of Molecular and Clinical Medicine, Ninewells Hospital and Medical School, University of Dundee, James Arrott Drive, Dundee DD2 1SY, Scotland, UK

E-mail: m.z.baxter@dundee.ac.uk

Abbreviations: CARG, Cancer and Aging Research Group; CGA, comprehensive geriatric assessment; CRASH, Chemotherapy Risk Assessment Scale for High-Age patients; ED, emergency department; ECOG, Eastern cooperative oncology group; EOX, epirubicin/oxaliplatin/capecitabine; FFS, failure-free survival; GOA, gastroesophageal adenocarcinoma; GORD, gastroesophageal reflux disease; GP, general practice; HR, hazard ratio; HRQoL, health-related quality of life; ICI, immune checkpoint inhibitor; LV function, left ventricular function; MDT, multi-disciplinary team; OS, overall survival; OTU, overall treatment utility; OX, oxaliplatin/capecitabine; PFS, progression-free survival; PS, performance status; RR, response rate; SACT, systemic anticancer therapy; UK, United Kingdom; 5FU, 5-fluorouracil

Key words: advanced gastroesophageal cancer, frailty, geriatric oncology, real-world, reduced performance status

\section{Contents}

1. Introduction

2. Patient experience and the role of the multi-disciplinary team

3. Treatment challenges in advanced gastroesophageal adenocarcinoma

4. Trial evidence for practice

5. Future direction

6. Conclusion

\section{Introduction}

The two major histological subtypes of gastroesophageal cancer are adenocarcinoma and squamous cell carcinoma. Gastroesophageal adenocarcinoma (GOA) is the most common histological subtype in developed countries and the incidence of GOA has markedly increased in the Western world in the last 40 years due to a rise in gastroesophageal reflux disease (GORD) and obesity (1).

GOA is a disease affecting older-aged individuals $(2,3)$. In the United Kingdom (UK), the median age at diagnosis is 74 years and there are approximately 15,000 cases diagnosed annually (2). As the population ages, this figure is projected to continue to rise, particularly in patients aged $\geq 80$ years (4).

Patients with GOA often have a high symptom burden and frequently have a reduced performance status and features of frailty $(3,5,6)$. It is therefore becoming crucial to understand how to best tailor treatments for the older-aged, frail patients with GOA in clinical practice.

Between 60 and $80 \%$ of patients with GOA present with advanced stage disease not amenable to curative treatment approaches with surgery or definitive chemoradiotherapy, or present when curative treatment is not possible due to medical co-morbidities or frailty $(7,8)$. The focus of treatment in these patients is disease control and the palliation of symptoms. There is ample evidence to suggest that systemic anticancer therapy in the form of chemotherapy can achieve this. Systemic treatment has also been shown to improve the health-related quality of life (9). However, at diagnosis, 
less than half of patients are assessed as being fit to receive palliative systemic therapy (10).

The median overall survival (OS) is approximately 3 months with the best supportive care $(9,11)$ and reported as 9-11 months with chemotherapy, extending to 16 months with the addition of trastuzumab to the subgroup (approximately $20 \%$ ) of patients whose tumours are human epidermal growth factor receptor 2 (HER2)-positive $(12,13)$. Emerging data suggest a role for immune checkpoint inhibitors (ICIs) in selected patients, which may provide more durable responses and result in a prolonged treatment duration, particularly in patients with an increased programmed death-ligand 1 (PD-L1) expression or mismatch repair deficiency $(14,15)$.

In those patients with advanced GOA who are deemed fit for systemic therapy, there is often a mismatch between their clinical phenotype and that observed in trial populations on which treatment guidelines are based. Clinicians are therefore required to apply information extrapolated from clinical trials in which patients are younger, fitter and have fewer comorbidities (16). This creates uncertainty around regime and dose due to concerns regarding the risk of toxicity with age-related changes to pharmacokinetics and pharmacodynamics. Ultimately this can result in the undertreatment of a patient and/or excess morbidity and mortality (17).

Despite this, trial data suggest that older-aged patients with advanced GOA can tolerate and benefit from systemic chemotherapy (18). As such, chronological age should not by itself preclude the use of anticancer therapy. Rather, a global picture of the patient's health should be considered, including functional status, comorbidities and social support. Frailty screening and comprehensive geriatric assessment (CGA) along with allied healthcare professional involvement should be part of this process (19).

The present narrative review discusses the challenges associated with the assessment of older-aged patients with advanced GOA and frailty that is commonly encountered in real-world clinical practice, the importance of frailty assessment and the current evidence which underpins our treatment decisions.

\section{Patient experience and the role of the multi-disciplinary team}

At diagnosis, the patient population with advanced GOA is characterised by an older age, a high symptom burden and a poor prognosis. For patients not fit to receive systemic treatment, the median OS is poor, estimated at 3 months (11). Contributing factors include the stage of disease and performance status; however, age does not appear to have an impact (18).

For patients who receive systemic therapy, there is a median OS benefit of approximately 6.7 months over supportive measures alone (20) and evidence to support improvement in symptoms (9). However, $>50 \%$ of patients require at least one treatment-related hospital admission, and $96 \%$ report fatigue (21). In all patients, there is a fine balance between the palliation of symptoms, improving survival and the risk of toxicity negatively impacting the quality of life.

Due to the nature of the disease, patients of all ages have often had a period of nutritional deficit. This, coupled with the high catabolic state of advanced disease, can result in malnutrition, immunodeficiency, an impaired quality of life and worse clinical outcomes (22). The impact is more evident in older-aged patients where age-related nutritional consequences, such as sarcopenia and osteoporosis are more common (23) and where other comorbidities are prevalent (24). In adults aged $\geq 60$, the prevalence of malnutrition varies according to the setting, estimated at $3.1 \%$ in the community to $28.7 \%$ in long-term care (25). This has important implications when assessing a patient's suitability for treatment. The treatment of advanced GOA therefore requires a multidisciplinary approach (Table I) with specific focus on input from palliative care, physicians with geriatric expertise (if available), general practice (GP), dietetics, occupational therapy and physiotherapy (26).

The medical team. Patients experience a high symptom burden in their final year of life (5). Symptoms related to tiredness, well-being and appetite become more severe earlier in the disease course, whereas symptoms related to drowsiness, pain and shortness of breath become more severe closer to death (5). The impact of symptom burden on both patients and the health service, was demonstrated by a study in Scotland, which found that $75 \%$ of patients with upper gastrointestinal cancer use GP out-of-hours services in the last year of life with a further $7.8 \%$ using hospital emergency departments (EDs) and 22.6\% using both GPs and EDs (27). In addition, patients with advanced GOA who survive $<7$ months following diagnosis, have an association with several indicators of low-quality end-of-life care (28).

Outpatient palliative care initiation has been shown to be associated with a decrease in symptoms and early palliative care involvement improves the quality of life at the end stages of life and reduces hospital visits (29). This highlights the importance of early cross-speciality collaboration.

Allied healthcare professionals. Cancer and its treatment can have an impact on a number of domains of a patient's health. It is therefore essential to assess for and address any functional, social or cognitive deficits early in the patient cancer journey. This can be done using frailty screening tools and a CGA, which will be discussed in detail below.

An example is the impact of cancer on the metabolic state of a patient, exacerbated in GOA by dysphagia. Systemic therapy can compound this further by causing symptoms, such as nausea, vomiting, mucositis and diarrhoea. The consequence is malnutrition, which in turn can influence the effectiveness of chemotherapy (30). A proportion of patients will require stent insertion to maintain oral intake, but all patients should receive regular nutritional assessments and dietetics input, as this has been shown to improve the quality of life and outcomes (31). In those who undergo stent insertion, close nutritional observation is required due to post stent pain.

Physical activity following a diagnosis of advanced cancer has the potential to prevent or reverse functional decline, control cancer-related symptoms and help maintain independence, thus improving outcomes (32). Older-aged patients with cancer are at an increased risk of falls compared to the general population (33) and prior falls are associated with a risk of 
Table I. Key elements of a patient's cancer journey, which require regular re-evaluation.

\begin{tabular}{|c|c|}
\hline Factors & Elements \\
\hline $\begin{array}{l}\text { Aims/goals of } \\
\text { treatment }\end{array}$ & $\begin{array}{l}\text { Improved survival } \\
\text { Improved/maintained quality of life } \\
\text { Minimise toxicity } \\
\text { Improve symptoms }\end{array}$ \\
\hline Patient factors & $\begin{array}{l}\text { Symptoms } \\
\text { Disease burden } \\
\text { Performance status/fitness } \\
\text { Age } \\
\text { Frailty status } \\
\text { Nutritional status } \\
\text { Organ function }\end{array}$ \\
\hline Treatment options & $\begin{array}{l}\text { Chemotherapy } \\
\text { Radiotherapy } \\
\text { Targeted therapy } \\
\text { Immune checkpoint inhibitors } \\
\text { Clinical trial } \\
\text { Procedural e.g., stenting } \\
\text { Best supportive care }\end{array}$ \\
\hline MDT involvement & $\begin{array}{l}\text { Oncologist } \\
\text { Surgeons } \\
\text { Palliative care } \\
\text { Geriatrics } \\
\text { General practice } \\
\text { Allied healthcare professionals } \\
\text { Cancer nurse specialists }\end{array}$ \\
\hline
\end{tabular}

Goals of care, patient factors, MDT involvement and available treatment options interact to create a plan of management. MDT, multi-disciplinary team.

chemotherapy toxicity and survival $(34,35)$. Chemotherapy in advanced GOA can contribute to an increased risk of falls; for example, dehydration may result in orthostatic hypotension or neurotoxicity, thus affecting balance. The involvement of physiotherapy and occupational therapy at an early stage can reduce the risk of adverse outcomes by facilitating strength and balance training, implementing home exercise programmes and providing a home safety evaluation.

The review of a patient by allied health professionals prior to treatment is part of prehabilitation. Prehabilitation is now widely practiced prior to GOA cancer surgery with significant outcome benefits related to nutrition, length of stay and improved complication rates (36-38). However, evidence relating to treatment outcomes with prehabilitation in advanced GOA is limited. Nevertheless, it has been demonstrated that individualised exercise and nutritional programmes for patients with advanced GOA result in significant improvements in functional and symptomatic domains (39). It stands to reason that enabling these improvements will reduce frailty and therefore improve tolerance of treatment.

\section{Treatment challenges in advanced gastroesophageal adenocarcinoma}

General considerations. Central to the process of decision-making are the wishes of the individual patient. Recent reports by Cancer Research UK and from North America highlight the importance of clear communication regarding the risks of treatment, and also demonstrate that the majority of older-aged cancer patients value quality of life over length of life $(40,41)$. These studies included patients with advanced GOA and highlight the importance of a personalised approach to management.

With improvements in medical care, life expectancy is increasing worldwide. As a result of this changing demographic, there is an increasing proportion of cancers diagnosed in the $>65$ age group. Within the UK, a third of all cancer diagnoses and half of all cancer-related deaths occur in the $>75$ age group (42).

The evidence for systemic anticancer therapy (SACT) in older populations is often extrapolated from retrospective and subgroup analyses of clinical trials in younger patients. Caution therefore needs to be applied and consideration given to altered physiology and drug pharmacology. Age alone is not an exclusion from chemotherapy as agents appear equally efficacious regardless of age (43). However, age-related changes in pharmacokinetics and pharmacodynamics often lead to increased toxicity when older and frailer patients are treated with doses established from clinical trials in younger, less frail patients $(18,44)$. Treatment decisions must be made in the context of life expectancy.

Frailty. Frailty is defined as a state of increased vulnerability toward stressors due to a multisystem reduction in reserve capacity (45). It is linked to both chronological age and the presence of comorbidities, but is considered a distinct concept.

Frailty is common in cancer, both due to the association of cancer with certain comorbidities and the increasing age, but also as the cancer itself places a physiological strain on the health of an individual. There are different models of the concept of frailty and no single diagnostic test. This means it is not possible to state an exact prevalence of frailty in older-aged patients with cancer.

A systematic review in 2015 evaluated data from 2,916 participants in 20 studies of frailty in older cancer patients (6). Studies were included if they used one or more of the established frailty models (phenotype model, cumulative deficit model or CGA). They found that the median reported prevalence of frailty and pre-frailty was $42 \%(6-86 \%)$ and $43 \%(13-79 \%)$, respectively. Only a median of $32 \%(11-78 \%)$ were classified as fit. Importantly, few patients included in the review had GOA and there is therefore a paucity of data relating specifically to prevalence of frailty in advanced GOA. Patients deemed frail or pre-frail were at increased risk of chemotherapy toxicity and intolerance as well as all-cause mortality.

Consequently, screening for frailty prior to commencing therapy is a useful step to identify a population that is at higher risk for toxicity. This screening should prompt more detailed investigation of specific patient needs. This is usually done by CGA, which not only identifies issues but also involves the intervention and follow-up. 
Traditionally, oncologists have used the Eastern Cooperative Oncology Group (ECOG) performance status (PS) or the Karnofsky performance scale as measures of fitness and frailty. Although very quick to do, they were validated in younger populations and do not take into account contributing domains of frailty such as medications, comorbidity and cognition (34) or interuser variability (46). They have also been shown to be inferior to other frailty screening tools and therefore should not be used alone (47).

Due to time limitations in clinical practice, a number of screening tools for frailty have been developed and validated with a focus on identifying patients who require a more in-depth assessment in the form of a CGA.

The Clinical Frailty Scale (48) also known as the Rockwood Score, is the most commonly used tool in the UK due to its ease of use and availability. It is based on clinical assessment using knowledge of cognition, social support, comorbidity and function. The patient is assigned a score between one and nine based on activity, function and disability. Other screening tools used in a cancer setting are the Geriatric 8 (G8) (49), Vulnerable Elders Survey-13 (50), the abbreviated CGA (51) and the Groningen Frailty Indicator (GFI) (52).

A screening tool may prompt the implementation of a CGA. This is used to identify causes of frailty, and target interventions appropriately (53). It is a detailed process with interdisciplinary input, which assesses multiple domains to create a problem list and subsequent plan of management. Although it is a strong predictor of adverse events with chemotherapy (19), its use in clinical practice is limited by time-constraints and the need for a physician with geriatric expertise. The implementation of CGA in gastrointestinal cancer has been shown to improve tolerance to chemotherapy, reduce dose modifications and lower rates of grade 3-5 toxicity $(54,55)$. A CGA should therefore be a standard of care for patients with advanced GOA deemed to have features of frailty.

\section{Drug-related issues}

Age-related changes. Physiology changes with age. This can have an impact on drug pharmacokinetics and pharmacodynamics (56). The age-related decline in renal function, as well as liver volume and blood flow impact the excretion and metabolism of drugs. In gastroesophageal cancer, this can be compounded by reduced gastric motility and absorption. A further complication is the age-associated reduction in lean body weight and muscle mass, which can impact the volume of distribution of lipid-soluble drugs. Taken together, these factors render the older-aged population more prone to drug-related side-effects, particularly when symptom control becomes a priority at the end of life.

Polypharmacy. Patients with advanced GOA, prior to their diagnosis, will often have multiple co-morbidities with associated prescribed medications. A UK study in patients with advanced cancer found the median number of medications was 7 (57). In that study, the median age and PS were 73 years and 2 respectively, similar to the advanced GOA population which is encountered.

In the context of SACT, a number of regimes involve the prescription of drugs that are inherently toxic and have narrow therapeutic windows. In addition, regimes often have supportive medications adding to the medication burden.
This can potentially lead to poor adherence or inappropriate medication use (58). In advanced GOA, a good example of a common drug interaction is the reduction in the efficacy of capecitabine if co-prescribed with a proton pump inhibitor (59). This has been shown to impact both progression-free survival (PFS) and OS. It is therefore vital to rationalise medications whenever possible.

Nutritional support. Malnutrition is an important prognostic factor in all cancer patients. Approximately 10-20\% of cancer-related deaths can be predominantly attributed to malnutrition as a consequence of the cancer itself $(60,61)$. A poor nutritional state is associated with poorer outcomes, such as lower response rates and survival, increased toxicity and a reduced quality of life (62-64). An age $>70$ years is associated with a 2 -fold increased risk of cancer mortality and severe malnutrition a 2.5-fold increased risk (61).

Advanced GOA can significantly affect nutrition with dysphagia and anorexia being common symptoms. In a study on 1,000 cancer outpatients, the median percentage weight loss for oesophageal and gastric cancers was 15.9 and $11.0 \%$, respectively (65). In these patients, weight loss was associated with anorexia and was greater in those with more advanced disease and with compromised performance status.

The recognition and management of nutritional issues is of particular importance in older-aged patients as they are predisposed to age-related reductions in lean muscle mass (sarcopenia) (66), as well as reduced gastrointestinal absorption (67). There is an additional challenge of delineating normal age-related changes from pathological cancer-related changes. In advanced gastric cancer, there is evidence that malnutrition exists in over half of patients and that it is associated with greater stage, elevated inflammatory markers and significantly lower survival (68). Nutritional assessment and subsequent intervention have been observed to improve survival.

Chemotherapy risk assessment tools. Chemotherapy toxicity can have a significant impact on a patient's quality of life. Toxicity occurring in the first cycle of treatment can predict those patients who will develop severe toxicity (69). Toxicity can result in reduced survival as a consequence of reduced dose intensity. The ability to predict which patients are more likely to develop side effects would facilitate a proactive approach, either dose reduction or the initiation of supportive measures. The goal for clinicians is to maintain efficacy of treatment, while minimising the negative impact on quality of life.

In advanced GOA, in a standard of care epirubicin/oxaliplatin/capecitabine (EOX) arm of the REAL2 trial (13), $42 \%$ of patients required a dose reduction and $50 \%$ of patients required at least one dose delay due to treatment-related toxicity (13). In the $\mathrm{GO} 2$ trial in a frail population, even with a $60 \%$ dose, $86 \%$ of patients experienced some form of toxicity and $37 \%$ experienced grade $\geq 3$ toxicity (70).

At present, there is no validated chemotherapy toxicity tool specifically for advanced GOA. There are two tools in general use that have been validated in a range of solid and haematological malignancies, the Cancer and Aging Research Group (CARG) tool and Chemotherapy Risk Assessment Scale for High-Age patients (CRASH) score. The CARG tool and CRASH score were developed as tools to predict severe 
toxicity in older-aged patients $(34,71)$. They use baseline patient and treatment factors to calculate a risk score and are more predictive of toxicity than age or performance status.

Although validated (72), their use is limited in advanced GOA due to a lack of agreement in which factors are important, the wide range of chemotherapy regimes and settings included and the low numbers of GOA patients. These limitations, coupled with the knowledge that the GOA population differ from other tumour types, highlight the need for a toxicity prediction tool specific to advanced GOA.

\section{Trial evidence for practice}

There have been a limited number of oncology studies focused on an older, frailer population treated with chemotherapy. The majority of the data used in clinical practice comes from retrospective data collection or sub-analysis of larger trials which included older patients within their eligibility criteria (Table II). When applying these data, consideration should also be given to the end points used, the traditional endpoint of OS may be less appropriate in an older population. Other outcomes, such as quality of life, the maintenance of a functional status and cognition may be as relevant or even more relevant than survival (73).

First-line chemotherapy. In advanced GO cancer, a standard of care chemotherapy in younger patients with a good PS (PS 0-1) was established by the REAL2 trial (13). In that trial, the median age of the EOX arm was 62 years and $90 \%$ of patients had PS 0 or 1 . In patients with features of frailty and reduced PS, a standard of care was established by the recently presented GO2 trial (70).

Prior to the GO2 trial, there was a lack of data in elderly, frail patients. Several phase I/II studies were conducted in the 1990s/early 2000s to investigate the use of chemotherapy in older patients with gastric cancer. Overall response rates (RRs) ranged from 29 to $45 \%$, with median PFS 4.2-5 months (74-77).

The only large, randomised trial in advanced GO cancer was the COMBAT study (78). That study tested the addition of mitomycin $\mathrm{C}$ to infusional 5-fluorouracil (5FU), demonstrating no statistically significant advantage in the primary endpoint, failure-free survival (FFS). The median age was $72,32 \%$ were PS 2 and the median OS was 6.3 months.

A pooled analysis in 2006 of three UK clinical trials in advanced GO cancer compared outcomes in patients aged $>70$ years to younger patients (18). A total of 1,080 patients were included, of whom 257 (23.8\%) were $>70$ years of age. No significant differences in RR, survival or toxicity were observed. A further study by the North Central Cancer Treatment Group (NCCTG) in which 154 patients were aged $>65$ years, found that although OS and RR were similar, the rates of toxicity were higher than those compared to patients younger, $<65$ years of age (79).

In 2015, the Phase II TTD 08-02 study assessed the use of a triplet regime in the form of reduced dose docetaxel with oxaliplatin and capecitabine in 42 'sub-optimal' patients. These patients were defined as those with PS 2, weight loss $10-25 \%$ and/or aged $\geq 70$ years. Although the median OS was 13.4 months, the rate of grade 3-5 toxicity was $76 \%$, with 3
(7\%) patients suffering a sudden death (80). Due to the high toxicity, this regime has not been adopted.

These studies suggested that three drug platinum-based chemotherapy often provided an unequal balance of toxicity over efficacy for older and frailer patients. This opinion was reflected in guidelines for GOA (81), and despite the lack of randomised trial evidence oncologists commonly made empirical dose reduction to chemotherapy in those older and frailer patients for whom there was concern that they would not tolerate standard dose three drug regimens.

To address the question of appropriate dosing in a frailer population a Phase II feasibility study in the UK, 321GO, was undertaken (16). It concluded that it was feasible to recruit older and/or frail patients with advanced GO cancer to a randomised clinical trial and that OX (oxaliplatin/capecitabine) was the preferred regimen for further study. That study also supported the use of the novel composite endpoint, overall treatment utility (OTU), including both patient-reported and clinical indices. This was initially used in the FOCUS2 trials in colorectal cancer (82) and reflects whether either or both the patient and clinician were pleased with the decision to proceed with chemotherapy (Table III).

The subsequent GO2 trial (70) aimed to establish the optimum dose-intensity $(100,80$ or $60 \%)$ of two-drug OX palliative chemotherapy in patients who are considered unsuitable for triplet EOX chemotherapy. The goal was to achieve the best balance of cancer control, toxicity, patient convenience, acceptability and quality of life. That study also aimed to establish pre-treatment patient characteristics which predict for better or worse outcomes with chemotherapy at different dose intensities.

A total of 517 patients were recruited, and the population appeared representative of real-world experience, median age 76 years, $31 \%$ PS $\geq 2$ and $>50 \%$ classified as very frail. The $60 \%$ dose was found to be non-inferior to $100 \%$ dose [median OS, 7.6 vs. 7.5 months; hazard ratio (HR), 1.10] with reduced toxicity (grade $3+$ adverse events, 37 vs. 56\%) and better OTU (good OTU, 43 vs. 35\%). On sub-analysis, no group was found to benefit from the higher dose, and the fittest patients benefited most from dose reduction. Age and PS were not predictive of OTU; however, this may be due to higher numbers of fit elderly patients and unfit younger patients within the trial.

As a result of that trial, the new 'full dose' standard of care treatment for patients with advanced GOA and features of frailty is $60 \%$ of the dose of oxaliplatin and capecitabine used in the REAL2 trial. Of note, in a sub-study of the GO2 trial, which recruited 45 patients in whom there was doubt over the benefit of giving chemotherapy, there was no significant survival advantage for single drug capecitabine over best supportive care (median OS, 6.1 vs. 3.0 months; HR, 0.64; $\mathrm{P}=0.34)(11)$.

Subsequent chemotherapy. When disease progression occurs during or following first-line treatment, only approximately $50 \%$ of patients are fit to receive further therapy (83). These patients are often frail as a result of their disease progression (3). In this setting, fewer older-aged patients are offered further treatment compared to younger patients (84). Second line chemotherapy options for advanced GOA include irinotecan, docetaxel and weekly paclitaxel administration. 


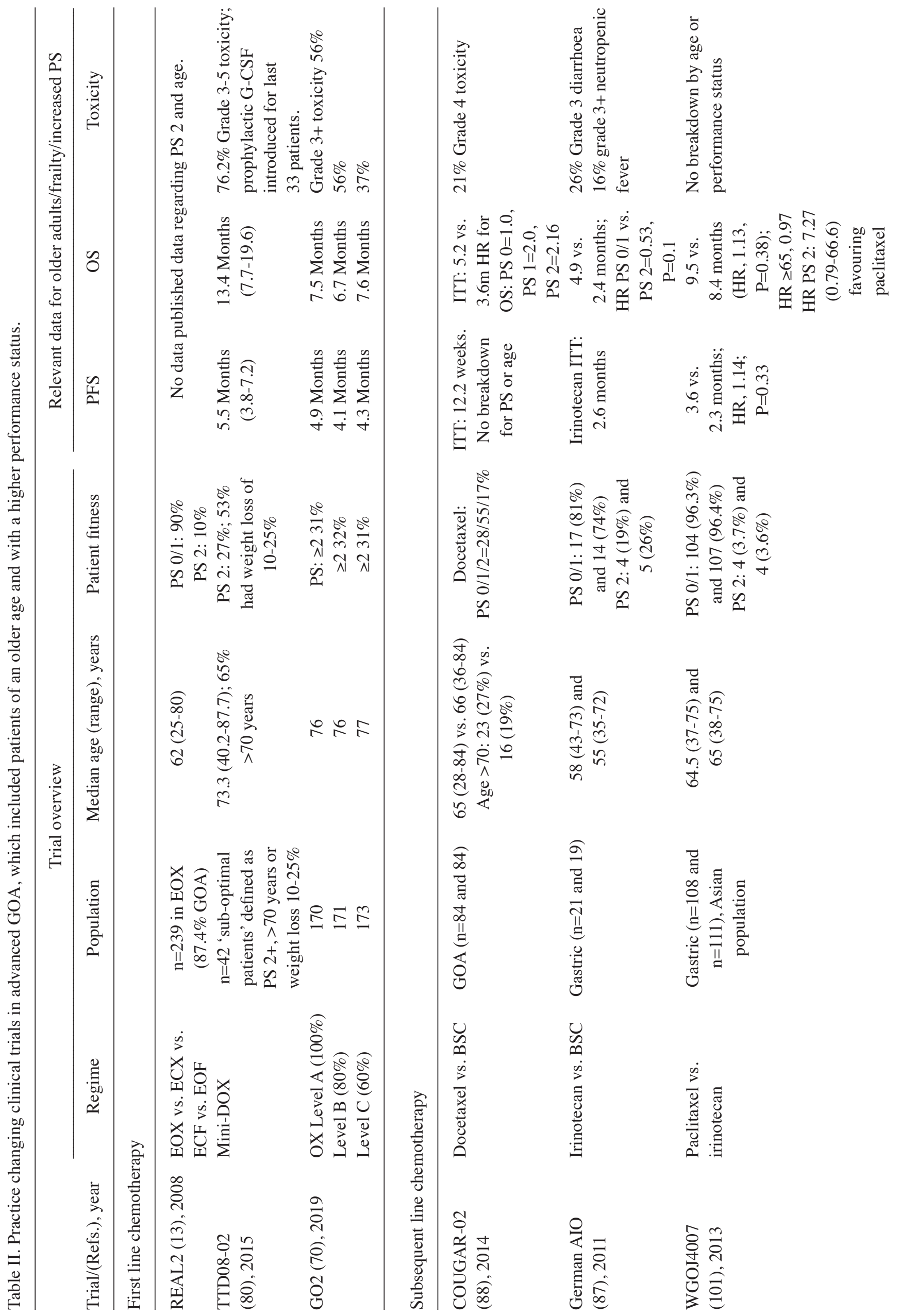




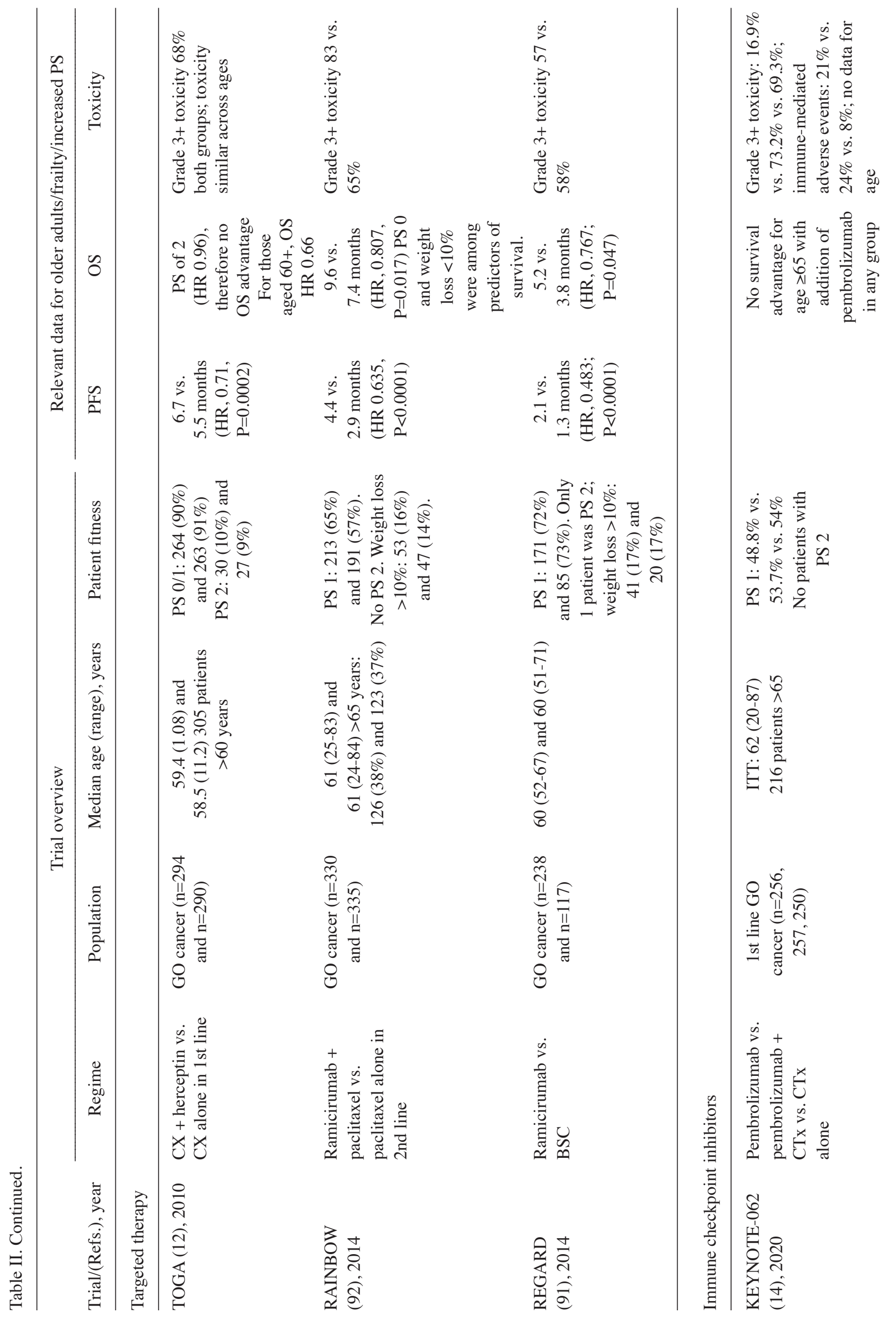


There is limited trial evidence for those who are older or of reduced performance status; however, extrapolated data exists that, for those who have PS 2 , and to a certain extent 1, the efficacy of further systemic therapy is minimal (85). In the second line COG study which did not stratify according to performance status, the median OS was 6.07, 3.93 and 1.97 months for patients with PS 0,1 and 2, respectively (86) highlighting the poor survival in those with a reduced PS.

The German AIO trial (87) and the COUGAR-02 trial (88) demonstrate efficacy from cytotoxic agents, such as irinotecan and docetaxel, respectively in the second line setting. However, the benefits of these treatments in the population as a whole were minimal and the toxicity was significant.

The German study of irinotecan included only 9 patients with a performance status of 2 . Despite this fact, these patients had a poorer outcome than those with PS0 or 1 (HR, 0.53). The COUGAR-02 trial included 39 patients aged $\geq 70$ and 26 patients with PS 2. Patients with a performance status of 0 had a better OS than those with a performance status of 1 [HR, 2.00; 95\% confidence interval (CI), 1.35-2.96] or 2 (HR, 2.16; 95\% CI, 1.27-3.66). On the whole, this supports the lack of efficacy of subsequent line therapy in frailer patients with a poor PS.

Beyond second line, the recently published TAGS trial (89) demonstrated a 2.1 month median OS advantage for lonsurf (trifluridine/tipiracil) when compared to supportive care. Lonsurf has now been licenced in the third line setting in the UK. However, only patients with PS 0 or 1 were recruited and the median age of patients recruited was 63 . The grade $3+$ toxicity rate was $53 \%$ and patients aged $>65$ who received Lonsurf $(\mathrm{n}=154)$, although trending for a survival advantage did not meet significance (HR, 0.73; 95\% CI, 0.52-1.02).

Targeted agents. In HER2-positive advanced GOA, following the results of the ToGA trial, there is the option to add trastuzumab to a chemotherapy backbone if left ventricular (LV) function permits (12). This is an important consideration in elderly patients, as LV function decreases with age (90). Within the ToGA trial, 57 patients had a PS of 2 and 305 patients were aged $>60$ years of age. There was no survival advantage for those with a PS of 2 (HR, 0.96), but a significant survival advantage for those aged $\geq 60$ years (HR, 0.66). This supports the hypothesis that age alone should not be used as the deciding factor in treatment decisions. Toxicity was similar according to age. Despite the low incidence of cardiac events within the trial, all patients require baseline cardiac imaging prior to starting trastuzumab in addition to ongoing monitoring.

Another licenced targeted agent is the vascular endothelial growth factor receptor (VEGFR)-2 monoclonal antibody, ramucirumab. The REGARD (91) trial demonstrated a survival benefit for ramucirumab over supportive care in the second line setting of 1.4 months. The majority of patients in the trial were performance status 1; only 1 patient was PS 2 and they did not receive the trial drug. However, given that clinicians were prepared to randomise to supportive care and only $35 \%$ of patients went on to receive subsequent therapy, PS may not provide an accurate representation of the frailty of the cohort. Therefore, although the survival benefit is minimal, the toxicity profile of ramucirumab is favourable, rendering this a more feasible option for subsequent therapy in frail patients, 
Table III. OTU scored after 9 weeks.

Good OTU
Intermediate OTU

Poor OTU
All of:

- Clinician score 'benefit'

- Patient satisfied

- No major toxicity

- No drop in QoL

\author{
Either: \\ - Clinician score 'no benefit' but patient \\ satisfied and no major toxicity or QoL drop \\ Or \\ - Either patient dissatisfied or major toxicity \\ or QoL drop, but clinician scores 'benefit'
}

Both:
- Clinician score 'no benefit'
And any of
- Patient dissatisfied
- Major toxicity
- QoL deterioration
Or

- Patient has passed away

Clinician scores 'benefit' indicates no clinical or radiological evidence of cancer progression. A drop in QoL is defined as a fall of $\geq 2$ on 12-point EORTC global QoL scale. Decision rules ensure OTU can be scored in 100\% of patients. OUT, overall treatment utility; QoL, quality of life. The table was provided by PSH.

particularly as the PFS and OS benefit was preserved for both PS and age. Of note, toxicity according to age was similar apart from an increased incidence of hypertension in the $>65$ age group.

The RAINBOW trial subsequently examined ramucirumab in addition to paclitaxel in the second line setting compared to paclitaxel alone (92). The addition of ramucirumab significantly improved OS (9.6 vs. 7.4 months, $\mathrm{P}=0.017)$ with only a minimal increase in toxicity. Again, only PS 0 or 1 patients were included but the PFS and OS was again preserved for PS and age. Of note, rates of neutropenia were higher for those aged $>65$. Taken together, the REGARD and RAINBOW trials provide an option for subsequent line therapy in those patients in whom there are concerns over toxicity.

Immune-checkpoint inhibitors. The advent of immune-checkpoint inhibitors has altered the treatment paradigm for several tumour groups. These agents have a more favourable toxicity profile than traditional chemotherapy in an older population with preserved efficacy (93).

Evidence for their use in advanced GOA is increasing; however, similar to trials with chemotherapy, there is a mismatch between real-world and trial populations. An example is the recent Keynote-061 trial in advanced GO cancer where no patients $>70$ years of age were included and the only patient with PS 2, did not receive the trial drug (94).

In the first-line setting, the Keynote-062 trial included no patients with PS 2 and $>50 \%$ had PS 0 (14). Of note, 216 patients were aged $\geq 65$ years and there did not appear to be a survival advantage in this group with the addition of pembrolizumab. Importantly, despite this apparently fit population, grade 3+ toxicity was $69 \%$ with chemotherapy and $73 \%$ with the addition of pembrolizumab.

Likewise, the CheckMate 649 trial did not include patients with PS 2 and the median age was 63 years. Of the patients included, 59\% had PS 1 and the median OS was 12.6 months, compared to 17.6 months in patients with PS 0. Again, grade $3+$ toxicity was high at $59 \%$ in the combination arm (15).

This highlights the challenge of combination therapy which will likely have important implications in real-world clinical practice.
Radiotherapy. The recently published ROCS study (95) investigated the role of palliative radiotherapy in patients who had had a self-expanding oesophageal stent inserted for dysphagia. The median age was 72 years in the radiotherapy group and only $10 \%$ of patients had PS 0 . No improvement in time to dysphagia deterioration or overall survival was observed with the addition of radiotherapy compared to the usual care group. However, for patients considered to be at high risk of bleeding, concurrent palliative radiotherapy may reduce bleeding risk.

Early supportive care. As mentioned above, advanced GOA is a high burden disease. This disease burden can impact not only on quality of life but also the tolerance of treatment. A recent study in China demonstrated that the early integration of nutritional and psychological support alongside SACT provided a survival advantage in advanced GO cancer (14.8 vs. 11.9 months; HR, 0.68; $\mathrm{P}=0.021)$ (96). No difference in frequency of adverse events was observed. Where available, early supportive care should therefore be offered to patients.

\section{Future direction}

For all patients, both cancer and the systemic treatments offered by oncologists are significant stressors that have the potential to challenge physiological reserve. For those patients with advanced disease, the delivery of treatment can be challenging, and the impact of treatment can be unpredictable and significant.

There is a recognised mismatch between the age of patients with advanced GOA encountered in clinical practice (median age, 74 years) and trial populations (REAL2 trial; median age, 63 years) (13). There is similar but less measurable mismatch in other measures such as frailty, performance status and co-morbidity. This issue is not unique to upper gastrointestinal cancer. The ageing population in the Western world can be expected to lead to a marked increase in the number of older patients seeking systemic anticancer therapy over the coming decades (97).

A lack of evidence in older-aged frail patients across tumour groups impacts negatively upon treatment delivery and effectiveness, as well as health-related quality of Life 
(HRQofL). These factors lead to uncertainty in selecting optimal regimens to achieve the best balance of cancer control, toxicity, patient acceptability and quality of life in older and frailer patients.

With the emerging role of immune checkpoint inhibitors and other targeted therapies in advanced GOA, it is important to include patients who represent our real-world patients in terms of age and frailty in prospective trials.

To address this issue, the inclusion of frailer patients in clinical trials, who more resemble those encountered in practice, as well as the incorporation of appropriate endpoints has been highlighted as a priority for the American Society of Clinical Oncology (ASCO), the International Society of Geriatric Oncology (SIOG) and the European Organization for Research and Treatment of Cancer (EORTC). They have produced reports on the topic (73,98-100) and have suggested removing the upper age limit of trials and the design of specific trials for older patients where standard therapy is not feasible with integrated appropriate measures of outcome.

Consideration should be given to identifying relevant clinical and translational questions and designing trials appropriately to address them. With an older, frailer population, these questions may involve issues relating to dose de-escalation to achieve a balance of efficacy and toxicity or validating novel endpoints. In the complex world of geriatric oncology where there is huge variation in patient fitness and circumstances, communication with patients and families is essential. The GO2 trial is an exemplar of this approach. The data from the GO2 trial offers the opportunity to develop a decision aid based on objective assessments of frailty and both clinical and patient reported outcomes.

In the absence of evidence from clinical trials, the role of real-world data should not be ignored. These data can provide insight into outcomes post-drug approval in patient groups not adequately represented in clinical trials. This can aid clinical decision making but also accelerate progress in developing appropriate future studies.

\section{Conclusion}

The real-world patient cohort of patients with advanced GOA differs from traditional trial populations. Palliative chemotherapy is effective in older and frail patients, but it is vital to monitor patients closely for toxicity. The proper selection of patients is paramount, and this highlights the importance of integration of frailty screening and geriatric assessment, multi-disciplinary team (MDT) input and toxicity prediction tools into decision making. In the future, there should be a move to make clinical trials more applicable to real-world populations.

\section{Acknowledgements}

Not applicable.

\section{Funding}

MAB is a clinical academic fellow funded by the Chief Scientist Office in Scotland.

\section{Availability of data and materials}

Not applicable.

\section{Authors' contributions}

MAB and SOH developed the concept for the study. MAB, SOH and RDP drafted the manuscript. RDP, PSH and DS reviewed and edited the manuscript. All authors read and approved the final draft. MAB and RDP confirm the authenticity of all the raw data.

\section{Ethics approval and consent to participate}

Not applicable.

\section{Patient consent for publication}

Not applicable.

\section{Competing interests}

MAB received funding from Servier and BMS to attend meetings. RDP has undertaken speaking, consulting and advisory roles for Eli Lilly, BMS, Pfizer, Sanofi and Servier, and received research funding (not related to the work in this manuscript) from Astra Zeneca, Roche, MSD, Merck serrano, Eli Lilly, Five Prime Therapeutics, Clovis, Boston Biomedical, and Janssen. PSH, DS and SOH have no competing interests to declare.

\section{References}

1. Edgren G, Adami HO, Weiderpass E and Nyrén O: A global assessment of the oesophageal adenocarcinoma epidemic. Gut 62: 1406-1414, 2013.

2. UK CR: Oesophageal Cancer, 2020.

3. Shitara K, Muro K, Matsuo K, Ura T, Takahari D, Yokota T, Sawaki A, Kawai H, Ito S, Munakata $M$ and Sakata Y: Chemotherapy for patients with advanced gastric cancer with performance status 2. Gastrointest Cancer Res 3: 220-224, 2009.

4. Offman J, Pesola F and Sasieni P: Trends and projections in adenocarcinoma and squamous cell carcinoma of the oesophagus in England from 1971 to 2037. Br J Cancer 118: 1391-1398, 2018.

5. Merchant SJ, Brogly SB, Booth CM, Goldie C, Nanji S, Patel SV, Lajkosz K and Baxter NN: Palliative care and symptom burden in the last year of life: A population-based study of patients with gastrointestinal cancer. Ann Surg Oncol 26: 2336-2345, 2019.

6. Handforth C, Clegg A, Young C, Simpkins S, Seymour MT, Selby PJ and Young J: The prevalence and outcomes of frailty in older cancer patients: A systematic review. Ann Oncol 26: 1091-1101, 2015.

7. Besharat S, Jabbari A, Semnani S, Keshtkar A and Marjani J: Inoperable esophageal cancer and outcome of palliative care. World J Gastroenterol 14: 3725-3728, 2008.

8. Fontana E and Smyth EC: Novel targets in the treatment of advanced gastric cancer: A perspective review. Ther Adv Med Oncol 8: 113-125, 2016.

9. Murad AM, Santiago FF, Petroianu A, Rocha PR, Rodrigues MA and Rausch M: Modified therapy with 5-fluorouracil, doxorubicin, and methotrexate in advanced gastric cancer. Cancer 72 : 37-41, 1993.

10. Partnership HQI: National Oesophago-Gastric Cancer Audit-An audit of the care received by people with oesophago-gastric cancer and oesophageal high grade dysplasia in England and Wales, 2019. 
11. Swinson D, Hingorani M,Stokes Z,Dent J, Guptal K, Chatterjee A Kamposioras K, Grumett SA, Khan M, Marshall H, et al: Best supportive care (BSC) with or without low-dose chemotherapy (chemo) in frail elderly patients with advanced gastroesophagea cancer (aGOAC): The uncertain randomization of the GO2 phase III trial. J Clin Oncol 37: 4051-4051, 2019.

12. Bang YJ, Van Cutsem E, Feyereislova A, Chung HC, Shen L, Sawaki A, Lordick F, Ohtsu A, Omuro Y, Satoh T, et al: Trastuzumab in combination with chemotherapy versus chemotherapy alone for treatment of HER2-positive advanced gastric or gastro-oesophageal junction cancer (ToGA): A phase 3, open-label, randomised controlled trial. Lancet 376: 687-697, 2010.

13. Cunningham D, Starling N, Rao S, Iveson T, Nicolson M, Coxon F, Middleton G, Daniel F, Oates J and Norman AR; Upper Gastrointestinal Clinical Studies Group of the National Cancer Researc: Capecitabine and oxaliplatin for advanced esophagogastric cancer. N Engl J Med 358: 36-46, 2008

14. Shitara K, Van Cutsem E, Bang YJ, Fuchs C, Wyrwicz L, Lee KW, Kudaba I, Garrido M, Chung HC, Lee J, et al: Efficacy and safety of pembrolizumab or pembrolizumab plus chemotherapy vs chemotherapy alone for patients with first-line, advanced gastric cancer: The KEYNOTE-062 phase 3 randomized clinical trial JAMA Oncol 6: 1571-1580, 2020.

15. Moehler M, Shitara S, Garrido M, Salman P, Shen L, Wyrwicz L, Yamaguchi K, Skoczylas T, Campos Bragagnoli A, Liu T, et al: LBA6_PR Nivolumab (nivo) plus chemotherapy (chemo) versus chemo as first-line (1L) treatment for advanced gastric cancer/gastroesophageal junction cancer (GC/GEJC)/esophageal adenocarcinoma (EAC): First results of the CheckMate 649 study. Ann Oncol 31 (Suppl 4): S1191, 2020.

16. Hall PS, Lord SR, Collinson M, Marshall H, Jones M, Lowe C, Howard H, Swinson D, Velikova G, Anthoney A, et al: A randomised phase II trial and feasibility study of palliative chemotherapy in frail or elderly patients with advanced gastroesophageal cancer (321GO). Br J Cancer 116: 472-478, 2017.

17. Swaminathan D and Swaminathan V: Geriatric oncology: Problems with under-treatment within this population. Cancer Biol Med 12: 275-283, 2015.

18. Trumper M, Ross P, Cunningham D, Norman AR, Hawkins R, Seymour M, Harper P, Iveson T, Nicolson M and Hickish T: Efficacy and tolerability of chemotherapy in elderly patients with advanced oesophago-gastric cancer: A pooled analysis of three clinical trials. Eur J Cancer 42: 827-834, 2006

19. Wildiers H, Heeren P, Puts M, Topinkova E, Janssen-Heijnen ML, Extermann M, Falandry C, Artz A, Brain E, Colloca G, et al: International society of geriatric oncology consensus on geriatric assessment in older patients with cancer. J Clin Oncol 32: 2595-2603, 2014

20. Wagner AD, Syn NL, Moehler M, Grothe W, Yong WP, Tai BC, Ho J and Unverzagt S: Chemotherapy for advanced gastric cancer. Cochrane Database Syst Rev 8: CD004064, 2017.

21. Waddell T, Chau I, Cunningham D, Gonzalez D, Okines AF, Okines C, Wotherspoon A, Saffery C, Middleton G, Wadsley J, et al: Epirubicin, oxaliplatin, and capecitabine with or without panitumumab for patients with previously untreated advanced oesophagogastric cancer (REAL3): A randomised, open-label phase 3 trial. Lancet Oncol 14: 481-489, 2013.

22. Arends J: Struggling with nutrition in patients with advanced cancer: Nutrition and nourishment-focusing on metabolism and supportive care. Annals of Oncology 29 (Suppl 2): ii27-ii34, 2018.

23. Amarya S, Singh K and Sabharwal M: Changes during aging and their association with malnutrition. J Clin Gerontol Geriat 6 : 78-84, 2015.

24. Barnett K, Mercer SW, Norbury M, Watt G, Wyke S and Guthrie B: Epidemiology of multimorbidity and implications for health care, research, and medical education: A cross-sectional study. Lancet 380: 37-43, 2012

25. Cereda E, Pedrolli C,Klersy C, Bonardi C, Quarleri L, Cappello S, Turri A, Rondanelli M and Caccialanza R: Nutritional status in older persons according to healthcare setting: A systematic review and meta-analysis of prevalence data using $\mathrm{MNA}^{(\circledR)}$. Clin Nutr 35: 1282-1290, 2016.

26. Boniface MM, Wani SB, Schefter TE, Koo PJ, Meguid C, Leong S, Kaplan JB, Wingrove LJ and McCarter MD: Multidisciplinary management for esophageal and gastric cancer. Cancer Manag Res 8: 39-44, 2016.

27. Mills S, Buchanan D, Guthrie B, Donnan P and Smith B: Factors affecting use of unscheduled care for people with advanced cancer: A retrospective cohort study in Scotland. Br J Gen Pract 69: e860-e868, 2019.
28. Dalhammar K, Malmström M, Schelin M, Falkenback D and Kristensson J: The impact of initial treatment strategy and survival time on quality of end-of-life care among patients with oesophageal and gastric cancer: A population-based cohort study. PLoS One 15: e0235045, 2020.

29. Srivastava P: The benefits of early integration of palliative care as a part of standard outpatient oncology care. J Clin Oncol 32: 18, 2014

30. Vandebroek AJV and Schrijvers D: Nutritional issues in anti-cancer treatment. Ann Oncol 19: v52-v55, 2008.

31. Laviano A, Di Lazzaro L and Koverech A: Nutrition support and clinical outcome in advanced cancer patients. Proc Nutr Soc 77: 388-393, 2018.

32. Eyigor S and Akdeniz S: Is exercise ignored in palliative cancer patients? World J Clin Oncol 5: 554-559, 2014

33. Wildes TM, Dua P, Fowler SA, Miller JP, Carpenter CR, Avidan MS and Stark S: Systematic review of falls in older adults with cancer. J Geriatr Oncol 6: 70-83, 2015 .

34. Hurria A, Togawa K, Mohile SG, Owusu C, Klepin HD, Gross CP, Lichtman SM, Gajra A, Bhatia S, Katheria V, et al: Predicting chemotherapy toxicity in older adults with cancer: A prospective multicenter study. J Clin Oncol 29: 3457-3465, 2011.

35. Wildes TM, Ruwe AP, Fournier C, Gao F, Carson KR, Piccirillo JF, Tan B and Colditz GA: Geriatric assessment is associated with completion of chemotherapy, toxicity, and survival in older adults with cancer. J Geriatr Oncol 4: 227-234, 2013.

36. Doganay E and Moorthy K: Prehabilitation for esophagectomy. J Thorac Dis 11 (Suppl 5): S632-S638, 2019.

37. Faithfull S, Turner L, Poole K, Joy M, Manders R, Weprin J, Winters-Stone K and Saxton J: Prehabilitation for adults diagnosed with cancer: A systematic review of long-term physical function, nutrition and patient-reported outcomes. Eur J Cancer Care (Engl) 28: e13023, 2019.

38. Minnella EM, Awasthi R, Loiselle SE, Agnihotram RV, Ferri LE and Carli F: Effect of exercise and nutrition prehabilitation on functional capacity in esophagogastric cancer surgery: A randomized clinical trial. JAMA Surg 153: 1081-1089, 2018.

39. Hall CC, Cook J, Maddocks M, Skipworth RJE, Fallon M and Laird BJ: Combined exercise and nutritional rehabilitation in outpatients with incurable cancer: A systematic review. Support Care Cancer 27: 2371-2384, 2019.

40. Cancer Research UK: Improving Cancer Treatment and Care for an Ageing Population. Cancer Research UK, London, 2018. https://www.cancerresearchuk. org/sites/default/files/advancing_care_advancing_years_full_ report.pdf. Accessed November 4, 2020.

41. Soto-Perez-de-Celis E, Li D, Sun CL, Kim K, Twardowski P, Fakih M, Chung VM, Cristea CM, Lim D, Yuan Y, et al: Patient-defined goals and preferences among older adults with cancer starting chemotherapy (CT). J Clin Oncol 36: 10009, 2018.

42. Cancer Research UK: Cancer mortality by age.https://www.cancerresearchuk.org/health-professional/cancer-statistics/mortality/age. Accessed November 3, 2020.

43. Popescu RA, Norman A, Ross PJ, Parikh B and Cunningham D: Adjuvant or palliative chemotherapy for colorectal cancer in patients 70 years or older. J Clin Oncol 17: 2412-2418, 1999.

44. Al-Batran SE, Pauligk C, Homann N, Hartmann JT, Moehler M, Probst S, Rethwisch V, Stoehlmacher-Williams J, Prasnikar N, Hollerbach S, et al: The feasibility of triple-drug chemotherapy combination in older adult patients with oesophagogastric cancer: A randomised trial of the arbeitsgemeinschaft internistische onkologie (FLOT65+). Eur J Cancer 49: 835-842, 2013.

45. Morley JE, Vellas B, van Kan GA, Anker SD, Bauer JM, Bernabei R, Cesari M, Chumlea WC, Doehner W, Evans J, et al: Frailty consensus: A call to action. J Am Med Dir Assoc 14: 392-397, 2013.

46. Kelly CM and Shahrokni A: Moving beyond karnofsky and ECOG performance status assessments with New technologies. J Oncol 2016: 6186543, 2016.

47. Kirkhus L, Šaltytè Benth J, Rostoft S, Grønberg BH, Hjermstad MJ, Selbæk G, Wyller TB, Harneshaug M and Jordhøy MS: Geriatric assessment is superior to oncologists clinical judgement in identifying frailty. Br J Cancer 117: 470-477, 2017.

48. Rockwood K, Song X, MacKnight C, Bergman H, Hogan DB, McDowell I and Mitnitski A: A global clinical measure of fitness and frailty in elderly people. CMAJ 173: 489-495, 2005.

49. Soubeyran P, Bellera CA, Gregoire F, Blanc J, Ceccaldi J, Mertens CBB, Mathoulin-Pélissier S, Fonck M and Rainfray M: Validation of a screening test for elderly patients in oncology. J Clin Oncol 26: 20568-20568, 2008. 
50. Saliba D, Elliott M, Rubenstein LZ, Solomon DH, Young RT, Kamberg CJ, Roth C, MacLean CH, Shekelle PG, Sloss EM and Wenger NS: The vulnerable elders survey: A tool for identifying vulnerable older people in the community. J Am Geriatr Soc 49: 1691-1699, 2001.

51. Overcash JA, Beckstead J, Extermann M and Cobb S: The abbreviated comprehensive geriatric assessment (aCGA): A retrospective analysis. Crit Rev Oncol Hematol 54: 129-136, 2005.

52. Steverink N, Slaets J, Schuurmans H and Lis M: Measuring frailty: Developing and testing the GFI (Groningen Frailty Indicator). Gerontologist 41: 236-237, 2001

53. Rubenstein LZ, Stuck AE, Siu AL and Wieland D: Impacts of geriatric evaluation and management programs on defined outcomes: Overview of the evidence. J Am Geriatr Soc 39 (Suppl): 8S-18S, 1991.

54. Li D, Sun CL, Kim H, Chung V, Koczywas M, Fakih M, Chao J, Chien L, Charles K, dos Hughes SFS, et al: Geriatric assessment-driven intervention (GAIN) on chemotherapy toxicity in older adults with cancer: A randomized controlled trial. J Clin Oncol 38: 12010, 2020.

55. Kalsi T, Babic-Illman G, Ross PJ, Maisey NR, Hughes S, Fields P, Martin FC, Wang Y and Harari D: The impact of comprehensive geriatric assessment interventions on tolerance to chemotherapy in older people. Br J Cancer 112: 1435-1444, 2015.

56. Mangoni AA and Jackson SH: Age-related changes in pharmacokinetics and pharmacodynamics: Basic principles and practical applications. Br J Clin Pharmacol 57: 6-14, 2004.

57. Cashman J, Wright J and Ring A: The treatment of co-morbidities in older patients with metastatic cancer. Support Care Cancer 18 651-655, 2010.

58. Steinman MA and Hanlon JT: Managing medications in clinically complex elders: 'There's got to be a happy medium' JAMA 304: 1592-1601, 2010.

59. Chu MP, Hecht JR, Slamon D, Wainberg ZA, Bang YJ, Hoff PM, Sobrero A, Qin S, Afenjar K, Houe V, et al: Association of proton pump inhibitors and capecitabine efficacy in advanced gastroesophageal cancer: Secondary Analysis of the TRIO-013/LOGiC Randomized Clinical Trial. JAMA Oncol 3: 767-773, 2017

60. Mislang AR, Di Donato S, Hubbard J, Krishna L, Mottino G, Bozzetti F and Biganzoli L: Nutritional management of older adults with gastrointestinal cancers: An International society of geriatric oncology (SIOG) review paper. J Geriatr Oncol 9: 382-392, 2018

61. Pressoir M, Desné S, Berchery D, Rossignol G, Poiree B Meslier M, Traversier S, Vittot M, Simon M, Gekiere JP, et al: Prevalence, risk factors and clinical implications of malnutrition in french comprehensive cancer centres. Br J Cancer 102 966-971, 2010

62. Andreyev HJN, Norman AR, Oates J and Cunningham D: Why do patients with weight loss have a worse outcome when undergoing chemotherapy for gastrointestinal malignancies? Eur J Cancer 34: 503-509, 1998.

63. Ross P, Ashley S, Norton A, Priest K, Waters JS, Eisen T, Smith IE and O'Brien ME: Do patients with weight loss have a worse outcome when undergoing chemotherapy for lung cancers? Br J Cancer 90: 1905-1911, 2004.

64. Persson C and Glimelius B: The relevance of weight loss for survival and quality of life in patients with advanced gastrointestinal cancer treated with palliative chemotherapy. Anticancer Res 22: 3661-3668, 2002.

65. Bozzetti F and SCRINIO Working Group: Screening the nutritional status in oncology: A preliminary report on 1,000 outpatients. Support Care Cancer 17: 279-284, 2009.

66. Doherty TJ: Invited Review: Aging and sarcopenia. J Appl Physiol (1985) 95: 1717-1727, 2003.

67. Rémond D, Shahar DR, Gille D, Pinto P, Kachal J, Peyron MA, Dos Santos CN, Walther B, Bordoni A, Dupont D, et al: Understanding the gastrointestinal tract of the elderly to develop dietary solutions that prevent malnutrition. Oncotarget 6: 13858-13898, 2015.

68. Qiu M, Zhou YX, Jin Y, Wang ZX, Wei XL, Han HY, Ye WF, Zhou ZW, Zhang DS, Wang FH, et al: Nutrition support can bring survival benefit to high nutrition risk gastric cancer patients who received chemotherapy. Support Care Cancer 23: 1933-1939, 2015

69. Hurria A, Brogan K, Panageas KS, Jakubowski A, Zauderer M, Pearce C, Norton L, Howard J and Hudis C: Change in cycle 1 to cycle 2 haematological counts predicts toxicity in older patients with breast cancer receiving adjuvant chemotherapy. Drugs Aging 22: 709-715, 2005.
70. Hall PS, Swinson D, Waters JS, Wadsley J, Falk S, Roy R, Tillett T, Nicoll J, Cummings S, Grumett SA, et al: Optimizing chemotherapy for frail and elderly patients (pts) with advanced gastroesophageal cancer (aGOAC): The GO2 phase III trial. J Clin Oncol 37: 4006, 2019.

71. Extermann M, Boler I, Reich RR, Lyman GH, Brown RH, DeFelice J, Levine RM, Lubiner ET, Reyes P, Schreiber FJ III and Balducci L: Predicting the risk of chemotherapy toxicity in older patients: The chemotherapy risk assessment scale for high-age patients (CRASH) score. Cancer 118: 3377-3386, 2012.

72. Zhang J, Liao X, Feng J, Yin T and Liang Y: Prospective comparison of the value of CRASH and CARG toxicity scores in predicting chemotherapy toxicity in geriatric oncology. Oncol Lett 18: 4947-4955, 2019.

73. Wildiers H, Mauer M, Pallis A, Hurria A, Mohile SG, Luciani A, Curigliano G, Extermann M, Lichtman SM, Ballman K, et al: End points and trial design in geriatric oncology research: a joint European organisation for research and treatment of cancer-Alliance for clinical trials in oncology-international society of geriatric oncology position article. J Clin Oncol 31: 3711-3718, 2013.

74. SantiniD,GrazianoF,Catalano V,Di Seri M,Testa E, Baldelli AM, Giordani P, La Cesa A, Spalletta B, Vincenzi B, et al: Weekly oxaliplatin, 5-fluorouracil and folinic acid (OXALF) as first-line chemotherapy for elderly patients with advanced gastric cancer: Results of a phase II trial. BMC Cancer 6: 125, 2006.

75. Lee JL, Kang YK, Kang HJ, Lee KH, Zang DY, Ryoo BY, Kim JG, Park SR, Kang WK, Shin DB, et al: A randomised multicentre phase II trial of capecitabine vs S-1 as first-line treatment in elderly patients with metastatic or recurrent unresectable gastric cancer. Br J Cancer 99: 584-590, 2008.

76. di Bartolomeo M, Bajetta E, de Braud F, Bochicchio AM, Gebbia V, Bozzetti F, Doci R, Bonfanti G and Cozzaglio L: Phase II study of the etoposide, leucovorin and fluorouracil combination for patients with advanced gastric cancer unsuitable for aggressive chemotherapy. Oncology 52: 41-44, 1995.

77. Cascinu S, Fedeli A and Catalano G: Etoposide, leucovorin, 5-fluorouracil and interferon alpha-2b in elderly gastric cancer patients: A pilot study. Cancer Chemother Pharmacol 34: 72-74, 1994.

78. Tebbutt NC, Norman A, Cunningham D, Iveson T, Seymour M, Hickish T, Harper P, Maisey N, Mochlinski K, Prior Y and Hill M: A multicentre, randomised phase III trial comparing protracted venous infusion (PVI) 5-fluorouracil (5-FU) with PVI 5-FU plus mitomycin $\mathrm{C}$ in patients with inoperable oesophago-gastric cancer. Ann Oncol 13: 1568-1575, 2002.

79. Jatoi A, Foster NR, Egner JR, Burch PA, Stella PJ, Rubin J, Dakhil SR, Sargent DJ, Murphy BR and Alberts SR: Older versus younger patients with metastatic adenocarcinoma of the esophagus, gastroesophageal junction, and stomach: A pooled analysis of eight consecutive North Central Cancer Treatment Group (NCCTG) trials. Int J Oncol 36: 601-606, 2010.

80. Rivera F, Massutí B, Salcedo M, Sastre J, Martínez Galán J, Valladares-Ayerbes M, Serrano R, García de Paredes ML, Manzano JL, Galán M, et al: Phase II trial of miniDOX (reduced dose docetaxel-oxaliplatin-capecitabine) in 'suboptimal' patients with advanced gastric cancer (AGC). TTD 08-02. Cancer Chemother Pharmacol 75: 319-324, 2015.

81. Smyth EC, Verheij M, Allum W, Cunningham D, Cervantes A and Arnold D; ESMO Guidelines Committee: Gastric cancer: ESMO clinical practice guidelines for diagnosis, treatment and follow-up. Ann Oncol 27 (Suppl 5): v38-v49, 2016.

82. Seymour MT, Thompson LC, Wasan HS, Middleton G, Brewster AE, Shepherd SF, O'Mahony MS, Maughan TS, Parmar M, Langley RE, et al: Chemotherapy options in elderly and frail patients with metastatic colorectal cancer (MRC FOCUS2): An open-label, randomised factorial trial. Lancet 377: 1749-1759, 2011.

83. Salati M, Di Emidio K, Tarantino V and Cascinu S: Second-line treatments: Moving towards an opportunity to improve survival in advanced gastric cancer? ESMO Open 2: e000206, 2017.

84. Sugimoto A, Nishida T, Osugi N, Takahashi K, Mukai K, Nakamatsu D, Matsubara T, Hayashi S, Yamamoto M, Nakajima S, et al: Prediction of survival benefit when deciding between chemotherapy and best supportive therapy in elderly patients with advanced gastric cancer: A retrospective cohort study. Mol Clin Oncol 10: 83-91, 2019.

85. Kawazoe A, Fukuoka S, Nakamura Y, Kuboki Y, Mikamoto Y, Shima H, Fujishiro N, Higuchi T, Wakabayashi M, Nomura S, et al: An open-label phase II study of lenvatinib plus pembrolizumab in patients with advanced gastric cancer (EPOC1706). J Clin Oncol 38: 374, 2020 
86. Dutton SJ, Ferry DR, Blazeby JM, Abbas H, Dahle-Smith A, Mansoor W, Thompson J, Harrison M, Chatterjee A, Falk S, et al: Gefitinib for oesophageal cancer progressing after chemotherapy (COG): A phase 3, multicentre, double-blind, placebo-controlled randomised trial. Lancet Oncol 15: 894-904, 2014.

87. Thuss-Patience PC, Kretzschmar A, Bichev D, Deist T, Hinke A, Breithaupt K, Dogan Y, Gebauer B, Schumacher G and Reichardt P: Survival advantage for irinotecan versus best supportive care as second-line chemotherapy in gastric cancer-a randomised phase III study of the Arbeitsgemeinschaft Internistische Onkologie (AIO). Eur J Cancer 47: 2306-2314, 2011.

88. Ford HE, Marshall A, Bridgewater JA, Janowitz T, Coxon FY, Wadsley J, Mansoor W, Fyfe D, Madhusudan S, Middleton GW, et al: Docetaxel versus active symptom control for refractory oesophagogastric adenocarcinoma (COUGAR-02): An open-label, phase 3 randomised controlled trial. Lancet Oncol 15: 78-86, 2014.

89. Shitara K, Doi T, Dvorkin M, Mansoor W, Arkenau HT, Prokharau A, Alsina M, Ghidini M, Faustino C, Gorbunova V, et al: Trifluridine/tipiracil versus placebo in patients with heavily pretreated metastatic gastric cancer (TAGS): A randomised, double-blind, placebo-controlled, phase 3 trial. Lancet Oncol 19: 1437-1448, 2018.

90. Parikh JD, Hollingsworth KG, Wallace D, Blamire AM and MacGowan GA: Normal age-related changes in left ventricular function: Role of afterload and subendocardial dysfunction. Int J Cardiol 223: 306-312, 2016.

91. Fuchs CS, Tomasek J, Yong CJ, Dumitru F, Passalacqua R, Goswami C, Safran H, Dos Santos LV, Aprile G, Ferry DR, et al: Ramucirumab monotherapy for previously treated advanced gastric or gastro-oesophageal junction adenocarcinoma (REGARD): An international, randomised, multicentre, placebo-controlled, phase 3 trial. Lancet 383: 31-39, 2014.

92. Wilke H, Muro K, Van Cutsem E, Oh SC, Bodoky G, Shimada Y, Hironaka S, Sugimoto N, Lipatov O, Kim TY, et al: Ramucirumab plus paclitaxel versus placebo plus paclitaxel in patients with previously treated advanced gastric or gastro-oesophageal junction adenocarcinoma (RAINBOW): A double-blind, randomised phase 3 trial. Lancet Oncol 15: 1224-1235, 2014.

93. Elias R, Morales J, Rehman Y and Khurshid H: Immune checkpoint inhibitors in older adults. Curr Oncol Rep 18: 47, 2016
94. Shitara K, Özgüroğlu M, Bang YJ, Di Bartolomeo M, Mandalà M, Ryu MH, Fornaro L, Olesiński T, Caglevic C, Chung HC, et al: Pembrolizumab versus paclitaxel for previously treated, advanced gastric or gastro-oesophageal junction cancer (KEYNOTE-061): A randomised, open-label, controlled, phase 3 trial. Lancet 392: 123-133, 2018.

95. Adamson D, Byrne A, Porter C, Blazeby J, Griffiths G, Nelson A, Sewell B, Jones M, Svobodova M, Fitzsimmons D, et al: Palliative radiotherapy after oesophageal cancer stenting (ROCS): A multicentre, open-label, phase 3 randomised controlled trial. Lancet Gastroenterol Hepatol: Feb 18, 2021 (Epub ahead of print).

96. Lu Z, Fang Y, Liu C, Zhang X, Xin X, He Y, Cao Y, Jiao X, Sun T, Pang Y, et al: Early interdisciplinary supportive care in patients with previously untreated metastatic esophagogastric cancer: A Phase III randomized controlled trial. J Clin Oncol 39 748-756, 2021.

97. Lord SR, Hall PS and Seymour MT: Are we representing the true population in oncology trials? Ann Oncol 20: 2022, 2009.

98. Hempenius L, Slaets JP, Boelens MA, van Asselt DZ, de Bock GH, Wiggers T and van Leeuwen BL: Inclusion of frail elderly patients in clinical trials: Solutions to the problems. J Geriatr Oncol 4: 26-31, 2013.

99. Whelehan S, Lynch O, Treacy N, Gleeson C, Oates A and O'Donovan A: Optimising clinical trial design in older cancer patients. Geriatrics (Basel) 3: 34, 2018

100. Hurria A, Levit LA, Dale W, Mohile SG, Muss HB, Fehrenbacher L, Magnuson A, Lichtman SM, Bruinooge SS, Soto-Perez-de-Celis E, et al: Improving the evidence base for treating older adults with cancer: American society of clinical oncology statement. J Clin Oncol 33: 3826-3833, 2015.

101. Hironaka S, Ueda S, Yasui H, Nishina T, Tsuda M, Tsumura T, Sugimoto N, Shimodaira H, Tokunaga S, Moriwaki T, et al: Randomized, open-label, phase III study comparing irinotecan with paclitaxel in patients with advanced gastric cancer without severe peritoneal metastasis after failure of prior combination chemotherapy using fluoropyrimidine plus platinum: WJOG 4007 trial. J Clin Oncol 31: 4438-4444, 2013.

This work is licensed under a Creative Commons Attribution-NonCommercial-NoDerivatives 4.0 International (CC BY-NC-ND 4.0) License. 